\title{
Sul numero delle falde delle superficie algebriche.
}

\author{
Nota di Margmerita Piazzolla Beioch (a Fertara).
}

Sunto. - Detta secondo KLEIN, falda di $1^{a}$ specie, ogni falda d'ordine pari contenente circwiti d'ordine dispari, l'A. dimostra che: una superficie algebrica d'ordine 2 n pari priva di singolarità, non può avere pì di $\frac{(2 \mathrm{n}-1)(2 \mathrm{n}-2)}{2}+1$ falde d'ordine pari di prima specie, e che esistono effettivamente, per ogni valore di $\mathrm{n}$, superficie algebriche d'ordine $2 \mathrm{n}$ per cui questo massimo è raggiunto. In questo caso la superficie non possiede falde ulteriori.

(Una superficie algebrica priva di singolaribła d'ordine dispari non possiede alcuna falda d'ordine pari di $1^{a}$ specie).

Secondo STAUDT: $\left.{ }^{(}\right)$le falde di una superficie algebrica si distinguono in falde d'ordine pari e d'ordine dispari, secondo che un piano generico dello spazio le seghi in un numero pari o dispari di punti reali. Le falde d'ordine pari si distinguono ulteriormente $\left({ }^{2}\right)$ in falde che contengono dei circuiti d'ordine dispari e falde che non ne contengono. Un esempio della prima specie di falde d'ordine pari ¿̀ dato dall'iperboloide rigato, un esempio della seconda specie di falde d'ordine pari è dato dall'ellissoide oppure dall'iperboloide ellittico.

Un circuito d'ordine dispari e una falda d'ordine dispari, che non si appartengano, hanno almeno un punto reale in comune. Da questa proprietà si deduce che due falde d'ordine dispari si segano necessariamente, come pure si segano una falda d'ordine dispari e una falda d'ordine pari di prima specie.

Segue che una superficie algebrica d' ordine $n$, priva di singolarità, per $n$ pari non possiede alcuna falda d'ordine dispari, per $n$ dispari ne possiede una e una sola. Segue ancora che una superficie d'ordine dispari priva di singolarità non può avere falde d'ordine pari di prima specie.

Ricordate queste proprietà, io nella presente Nota mi occupo delle falde d'ordine pari di prima specie e dimostro che una superficie algebrica F d'ordine $2 \mathrm{n}$, priva di singolarità, non pù̀ avere più di $\frac{(2 \mathrm{n}-1)(2 \mathrm{n}-2)}{2}+1$ falde d'ordine pari di prima specie. Esistono effettivamente, per ogni valore di $n$, superficie algebriche d'ordine $2 n$ per cui questo massimo è raggiunto. In tal caso anzi la superficie non pnò possedere falde ulteriori.

(1) Staudr, Geometrie der Lage (1847).

(") F. KLEIN, Flächen dritter Ordnung, \& 15, "Math. Annalen », Bd. 6 (1873) (551-581). 577; "Gesammelte Mathematische AbhandInngen", XXXV. 
Osserviamo che la superficie $F$, essendo d'ordine pari, potrà avere soltanto falde d'ordine pari (di prima o seconda specie) data J'ipotesi.

Dimostriamo in primo luogo, che il numero delle falde d'ordine pari di prima specie non puó superare $\frac{(2 n-1)(2 n-2)}{2}+1$.

Seghiamo la superficie $F$ con un piano generico. Questo piano sega ognuna delle falde d'ordine pari di prima specie della superficie in un circuito d'ordine pari reale, perchè nna falda di prima specie contiene circuiti dispari che segano ognuno necessariamente il piano almeno in un punto reale, e se un circuito piano d'ordine pari ha uno o più punti reali (semplici) ne contiene infiniti $\left({ }^{3}\right)$.

Ora, se $p$ è il genere della sezione pirna, per il teorema di HaRnack ( $\left.{ }^{4}\right)$, i circuiti che possiede sono al massimo $p+1$, ed altrettanto possono quindi essere al massimo le falde d'ordine pari di prima specie della superficie.

Ma, essendo $2 n$ l'ordine della sezione piana, sarà $p=\frac{(2 n-1)(2 n-2)}{2}$ e il numero $\delta$ delle falde di prima specie sarà al massimo $p$, ossia

$$
\delta \leq \frac{(2 n-1)(2 n-2)}{2}+1
$$

c.v.d.

Consideriamo il caso in cui questo massimo sia raggiunto.

Supponiamo che, oltre le $\delta$ falde d'ordine pari di prima specie, la superficie possegga un'altra falda, necessariamente d'ordine pari di seconda specie. Sia $\Gamma$ questa falda, supposta esistente. Per un punto situato sopra $\Gamma$ conduciamo un piano ad arbitrio. Questo segherà la falda $\Gamma$ secondo un circuito reale e segherà inoltre secondo circuiti reali tutte le $\delta$ falde d'ordine pari di prima specie. La curva d'intersezione, del piano con la superficie avrà dunque in tutto $\delta+1=p+2$ circuiti, mentre, per il teorema di HARNAOK, una curva di genere $p$ ne può avere al massimo $p+1$. Dunque la falda $\Gamma$ non può esistere, e possiamo concludere che, se una superficie d'ordine 2n pos* siede il massimo numero di falde d'ordine pari di prima specie essa non può possedere alcuna falda uteriore.

Dimostriamo ora che esistono effettivamenle superficie algebriche $d$ 'ordine $2 n$ possedenti esattamente $\frac{(2 n-1)(2 n-2)}{2}+1$ falde d'ordine pari di prima specie, e non aventi altre falde ulteriori.

(3) Infatti se $A$ è un punto reale (semplice) del circuito piano d'ordine pari, ogni retta condotta per $A$, dovendo incontrare il eircuito in un numero pari di punti reali, contiene almeno un altro punto reale del circnito.

(*) A. HaRNACK, Ueber die Vieltheiligiceit der ebenen algebraischen Curven, * Math. Annalen », Bd. 10 (1876), rp. 189-198. 
Consideriamo una curva piana $C$ d'ordine $2 n$, priva di singolarita, possedente il massimo numero di circuiti (tutti d'ordine pari), ossia $p+1=\frac{(2 n-1)(2 n-2)}{2}+1$, e sia $f$ un cilindro (d'ordine $\left.2 n\right)$ avente questa curva come curva base e possedente quindi tante falde d'ordine pari di prima specie (cilindriche), quanti sono i circuiti della curva $C$. Siano poi $g_{1}, g_{2}, \ldots g_{n}$, $n$ ellissoidi non seganti il cilindro $f$ in curve reali.

Siano $f=0, g_{1}=0, g_{2}=0, \ldots, g_{n}=0$ rispettivamente le equazioni del cilindro e degli ellissoidi saddetti e formiamo la combinazione lineare

$$
f+\lambda g_{1} g_{2} \ldots g_{n}=0
$$

che rappresenterà, per $\lambda$ sufficientemente picoola, una superficie d'ordine $2 n$ prossima al cilindro $f$, dotata cioè di $p+1$ falde d'ordine pari di prima specie prossime alle $p+1$ falde d'ordine pari di prima specie del cilindro. Abbiamo dunque costruito una superficie d'ordine $2 n$ avente esattamente $\frac{(2 n-1)(2 n-2)}{2}+1$ falde d'ordine pari di prima specie, ossia il massimo consentito dall' ordine e non avente altre falde ulteriori. 